\title{
Tiotropium attenuates IL-13-induced goblet cell metaplasia of human airway epithelial cells
}

\author{
Loes E M Kistemaker, ${ }^{1,2}$ Pieter S Hiemstra, ${ }^{3}$ I Sophie T Bos, ${ }^{1,2}$ Susanne Bouwman, ${ }^{1,2}$ \\ Maarten van den Berge, ${ }^{2,4}$ Machteld N Hylkema, ${ }^{2,5}$ Herman Meurs, ${ }^{1,2}$ \\ Huib A M Kerstjens, ${ }^{2,4}$ Reinoud Gosens ${ }^{1,2}$
}

- Additional material is published online only. To view please visit the journal online (http://dx.doi.org/10.1136/ thoraxjnl-2014-205731).

${ }^{1}$ Department of Molecular Pharmacology, University of Groningen, Groningen, The Netherlands ${ }^{2}$ GRIAC Research Institute, University Medical Center Groningen, University of Groningen, Groningen, The Netherlands

${ }^{3}$ Department of Pulmonology, Leiden University Medical

Center, Leiden,

The Netherlands

${ }^{4}$ Department of Pulmonary Diseases, University of Groningen, University Medical Center Groningen, Groningen, The Netherlands

${ }^{5}$ Department of Pathology and Medical Biology, University Medical Center Groningen, Groningen, The Netherlands

\section{Correspondence to}

Loes E M Kistemaker, Department of Molecular Pharmacology, University of Groningen, A Deusinglaan 1, Groningen 9713 AV,

The Netherlands;

l.e.m.kistemaker@rug.nl

Received 13 May 2014 Revised 29 April 2015 Accepted 30 April 2015 Published Online First 20 May 2015

\begin{abstract}
Background It has been shown that acetylcholine is both a neurotransmitter and acts as a local mediator, produced by airway cells including epithelial cells. In vivo studies have demonstrated an indirect role for acetylcholine in epithelial cell differentiation. Here, we aimed to investigate direct effects of endogenous nonneuronal acetylcholine on epithelial cell differentiation. Methods Human airway epithelial cells from healthy donors were cultured at an air-liquid interface (ALI). Cells were exposed to the muscarinic antagonist tiotropium (10 nM), interleukin (IL)-13 (1, 2 and $5 \mathrm{ng} / \mathrm{mL}$ ), or a combination of IL-13 and tiotropium, during or after differentiation at the ALI.
\end{abstract}

Results Human airway epithelial cells expressed all components of the non-neuronal cholinergic system, suggesting acetylcholine production. Tiotropium had no effects on epithelial cell differentiation after air exposure. Differentiation into goblet cells was barely induced after air exposure. Therefore, IL-13 $(1 \mathrm{ng} / \mathrm{mL})$ was used to induce goblet cell metaplasia. IL-13 induced MUC5ACpositive cells (5-fold) and goblet cells (14-fold), as assessed by histochemistry, and MUC5AC gene expression (105-fold). These effects were partly prevented by tiotropium (47-92\%). Goblet cell metaplasia was induced by IL-13 in a dose-dependent manner, which was inhibited by tiotropium. In addition, tiotropium reversed goblet cell metaplasia induced by 2 weeks of IL-13 exposure. IL-13 decreased forkhead box protein A2 (FoxA2) expression (1.6-fold) and increased FoxA3 (3.6-fold) and SAM-pointed domaincontaining ETS transcription factor (SPDEF) (5.2-fold) expression. Tiotropium prevented the effects on FoxA2 and FoxA3, but not on SPDEF.

Conclusions We demonstrate that tiotropium has no effects on epithelial cell differentiation after air exposure, but inhibits and reverses IL-13-induced goblet cell metaplasia, possibly via FoxA2 and FoxA3. This indicates that non-neuronal acetylcholine contributes to goblet cell differentiation by a direct effect on epithelial cells.

\section{BACKGROUND}

Acetylcholine has long been known as a classical neurotransmitter in the airways, released from parasympathetic nerve fibres. It induces bronchoconstriction and mucus secretion via muscarinic receptors. For this reason, anticholinergic therapy

\section{Key messages}

What is the key question?

- Does acetylcholine have a direct effect on epithelial cell differentiation, in particular, IL-13-induced goblet cell metaplasia?

\section{What is the bottom line?}

- Tiotropium attenuates IL-13-induced goblet cell metaplasia of human airway epithelial cells, suggesting a direct role for endogenous acetylcholine in epithelial cell differentiation.

\section{Why read on?}

- Although it is expected that tiotropium attenuates mucus hypersecretion in patients, there is little evidence for a direct role of acetylcholine in epithelial cell differentiation and here we provide evidence for such a role and investigate possible mechanisms involved.

anticholinergic agent, tiotropium, has proven effective for the chronic treatment of asthma. ${ }^{1} 2$

In addition, there is evidence that acetylcholine can also be synthesised and released from nonneuronal origins, acting as an autocrine and/or paracrine mediator on airway cells. ${ }^{3}$ In particular, airway epithelial cells have been shown to express relatively high levels of acetylcholine. ${ }^{4} 5$ Epithelial cells express enzymes to synthesise acetylcholine, including choline acetyltransferase (ChAT), and have also been shown to release non-neuronal acetylcholine via organic cation transporters. ${ }^{46}$

Epithelial cells play an important role in asthma. Epithelial differentiation is altered in the disease, as there is an increase in goblet cell numbers, leading to excessive mucus production and hence obstruction of the airways. ${ }^{7}$ IL-13 is the main driver of this response and has been shown to play a central role in asthma. From animal models it is known that interleukin (IL)-13 is sufficient to induce goblet cell hyperplasia, ${ }^{9}$ which can be reproduced in air-liquid interface (ALI) cell cultures in vitro. ${ }^{10} 11$ IL-13-induced MUC5 AC gene expression is mediated via a complex transcriptional network, in which expression of the repressor, forkhead box protein A2 (FoxA2), is reduced in has been used for many centuries for the treatment of obstructive airway diseases, and the long-acting response to STAT6 activation, whereas expression of SAM-pointed domain-containing ETS transcription

To cite: Kistemaker LEM, Themstra PS, Bos IST, et al. 
factor (SPDEF) and FoxA3 are increased and promote MUC5AC gene expression. $^{12} 13$

Interestingly, several studies have indirectly demonstrated a role for acetylcholine in epithelial cell differentiation and goblet cell metaplasia. Tiotropium, an anticholinergic drug, has been shown to inhibit ovalbumin-induced goblet cell metaplasia in guinea pigs and mice, ${ }^{14} 15$ an effect also observed after knockout of the $\mathrm{M}_{3}$ receptor in mice. ${ }^{16}$ Furthermore, the increased MUC5AC expression observed after allergen exposure was inhibited by tiotropium in guinea pigs and $\mathrm{M}_{3}$ knockout mice. ${ }^{14} 16$ Moreover, in patients with mild asthma, repeated challenges with methacholine induced epithelial cell proliferation and goblet cell metaplasia. ${ }^{17}$ Although these studies point to a critical role for acetylcholine in goblet cell metaplasia, it remains unresolved if this is via direct regulation of goblet cell differentiation of airway epithelial cells or via the indirect regulation of proinflammatory cells and cytokines that drive this response, since tiotropium also inhibited the inflammatory response in the animal studies described above.

Therefore, in this study, we investigated the role of endogenous non-neuronal acetylcholine in the differentiation of primary human airway epithelial (HAE) cells, differentiated at an ALI cell culture. We demonstrate that inhibition of endogenous acetylcholine by tiotropium has no effect on epithelial cell differentiation after air exposure, but inhibits and reverses IL-13-induced goblet cell metaplasia and MUC5AC expression, which might be mediated via FoxA2 and FoxA3.

\section{METHODS}

\section{Culture of HAE cells}

The immortal human bronchial epithelial cell line, 16HBE14o -, was kindly donated by Dr D C Gruenert, University of Vermont, Burlington, Vermont, USA. ${ }^{18}$ Cells were cultured as described previously ${ }^{19}$ and briefly described in the online supplementary data.

Primary HAE cells were obtained from lung transplant donors post mortem, from residual tracheal and main stem bronchial tissue, within 1-8 h after lung transplantation. Selection criteria for transplant donors are listed in the Eurotransplant guidelines and include the absence of primary lung disease, such as asthma and COPD, and no more than 20 pack years of smoking history. The material was collected in carbogenated Krebs-Henseleit buffer (composition in mM: $117.5 \mathrm{NaCl}, 5.6 \mathrm{KCl}, 1.18 \mathrm{MgSO}_{4}$, $2.5 \mathrm{CaCl}_{2}, 1.28 \mathrm{NaH}_{2} \mathrm{PO}_{4}, 25 \mathrm{NaHCO}_{3}$ and 5.5 glucose). Epithelial cells were isolated by enzymatic digestion, and cultured as described previously, ${ }^{11}$ which is also briefly explained in the online supplementary data and summarised in figure 1. Cells were differentiated for 2 weeks at the ALI. In the initial experiments, cells were exposed to tiotropium (provided by Boehringer Ingelheim) at a dose of $10 \mathrm{nM}$ to achieve $>99 \%$ receptor occupancy ${ }^{20}$ during the differentiation period. In subsequent experiments, cells were exposed to tiotropium and/or IL-13 (1-5 ng/mL; Peprotech, Rocky Hill, New Jersey, USA). In the final experiments, cells were exposed to IL-13 and/or tiotropium for 2 weeks, and thereafter exposed to IL-13 and tiotropium for 1 week (figure 1).

\section{RNA analysis}

Total RNA was extracted from freshly isolated airway epithelial cells, from cultured 16HBE14o- cells, and from HAE cells, after either submerged or ALI culture (HAE cells), using the RNeasy mini kit (Qiagen, Venlo, The Netherlands) according to the manufacturer's instructions. Equal amounts of total RNA were then reverse-transcribed, and cDNA was subjected to real-time

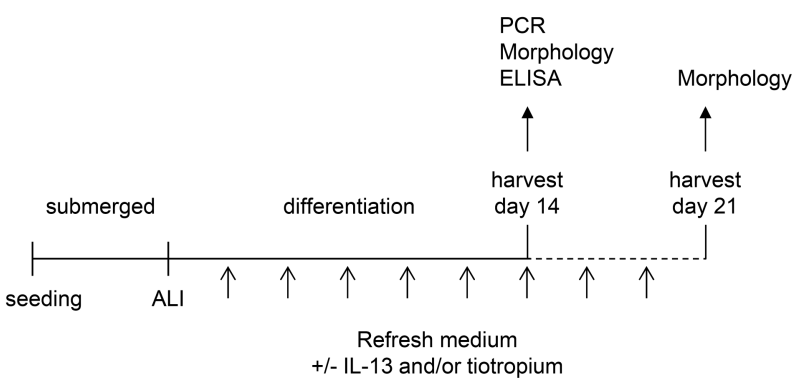

Figure 1 Schematic representation of the air-liquid interface (ALI) culture model. Human airway epithelial (HAE) cells were seeded on to a transwell insert and grown to confluence. Thereafter, apical medium was removed to create an ALI. Medium and stimuli were refreshed three times per week. Cells were harvested after 14 or 21 days for PCR analysis or morphology, and medium was collected for ELISA. IL, interleukin.

quantitative (q)PCR (Westburg, Leusden, The Netherlands) or to micro-array analysis. Details on real-time qPCR and primers used can be found in the online supplementary data (text and table E1). Data are normalised to $18 \mathrm{~S}$ ribosomal RNA. Micro-array analysis was performed on ALI-cultured cells from four different donors, exposed to vehicle or tiotropium, and analysed in duplicate. Assuming an SD of 0.5 , we had a power of $80 \%$ to detect a twofold difference at a nominal $p$ value of 0.05 . Micro-array analysis was performed using the llumina HT12 V.4 chip. Data were analysed using $\mathrm{R}$ software and subjected to gene set enrichment analysis using the Kyoto Encyclopaedia of Genes and Genomes (KEGG), Biocarta and Reactome definitions.

\section{Histochemistry}

The morphology of the cultures was assessed using light microscopy. After 2 weeks, transwell inserts were embedded in paraffin according to the manufacturer's instructions. Transverse crosssections $5 \mu \mathrm{m}$ thick were used for morphometric analyses. Cells were stained with H\&E (Sigma-Aldrich, Zwijndrecht, The Netherlands), MUC5AC-positive cells were stained with a MUC5AC antibody stain (Neomarkers, Fremont, California, USA), and mucin-producing goblet cells were stained with periodic acid Schiff's reagent (PAS; Sigma-Aldrich, Zwijndrecht, The Netherlands). Cells were counted in duplicate in a blinded fashion. FoxA2 was stained with a rabbit anti-FoxA2 antibody (Abcam, Cambridge, UK) using a horseradish peroxidase-linked goat anti-rabbit secondary antibody (Dako, Glostrup, Denmark) and diaminobenzidine (Sigma-Aldrich).

\section{Cytokine analysis}

Cytokine concentrations in the basal medium were determined by a Milliplex assay (Millipore, Billerica, Massachusetts, USA) on a Luminex 100 system using Starstation software (Applied Cytometry Systems, Sheffield, UK) according to the manufacturer's instructions. A screen for 26 cytokines was performed (see online supplementary data for complete list).

\section{Statistical analysis}

Data are presented as mean \pm SEM. Statistical differences between means were calculated using one-way or two-way analysis of variance where appropriate, followed by Newman-Keuls multiple comparison tests. Differences were considered significant at $\mathrm{p}<0.05$. 

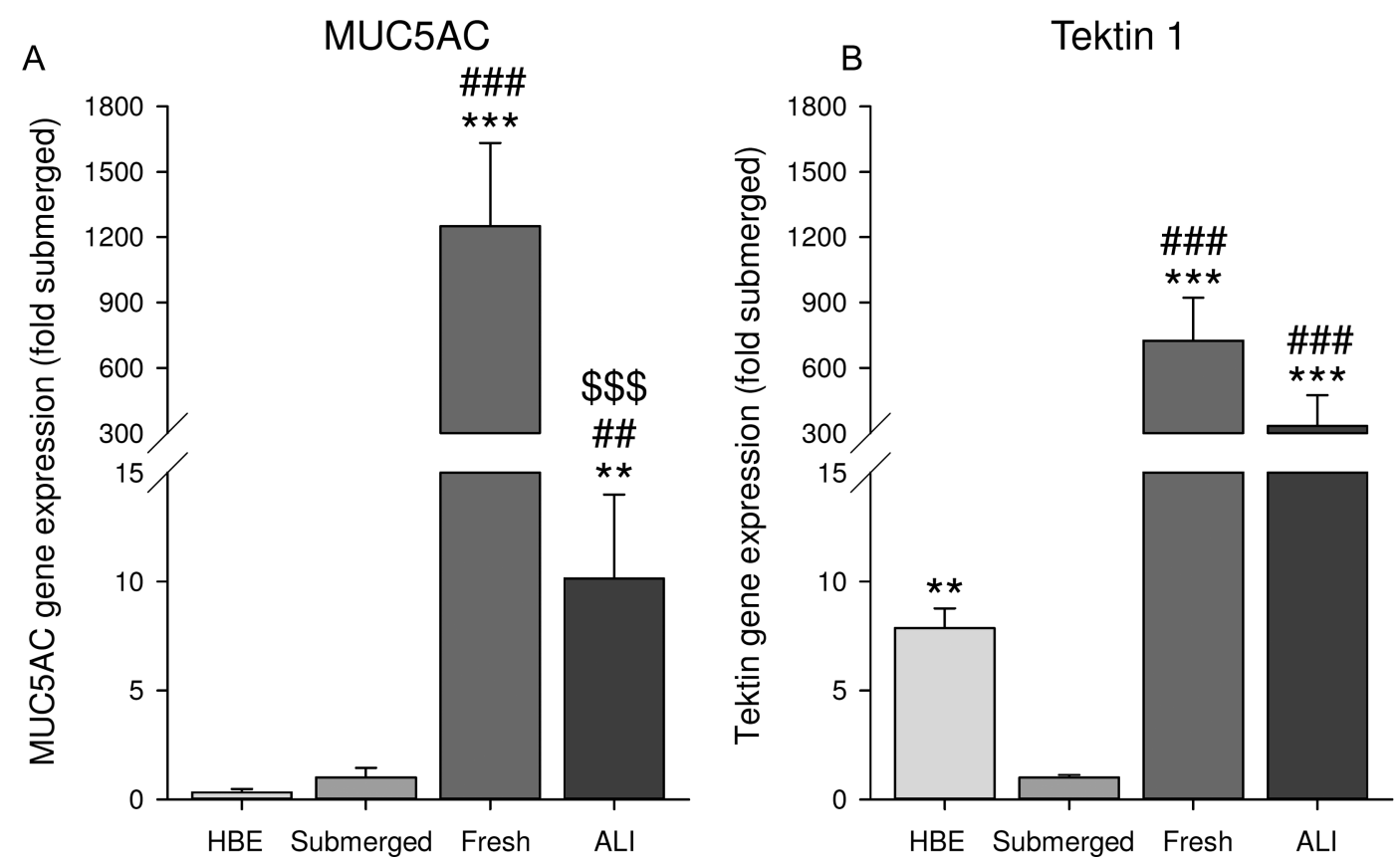

Figure 2 Differentiation state of epithelial cells. Expression of the differentiation markers, MUC5AC (a marker for goblet cells (A)) and tektin 1 (a marker for ciliated cells (B)), was analysed by real-time quantitative PCR in submerged 16HBE140 - cells and submerged human airway epithelial (HAE) cell cultures, in freshly isolated HAE cells and in ALI-cultured HAE cells. Data represent mean $\pm S E M, n=3-5 .{ }^{* *} p<0.01,{ }^{* * *} p<0.001$ compared with submerged culture; \#\#p $<0.01$, \#\#\# <0.001 compared with HBE culture; $\$ \$ \$ p<0.001$ compared with freshly isolated cells.

\section{RESULTS}

Differentiation of epithelial cells

First, the differentiation state of epithelial cells was characterised. Therefore, gene expression of the differentiation markers, MUC5AC (a marker for goblet cells) and tektin 1 (a marker for ciliated cells), was compared in different cells. We compared submerged cultures of the cell line 16HBE14o-, submerged cultures of HAE cells, freshly isolated HAE cells, and ALI-cultured HAE cells. Gene expression of MUC5AC and tektin 1 was higher in freshly isolated and ALI-cultured HAE cultures than in submerged cultures (figure 2). Whereas tektin 1 levels were comparable in freshly isolated cells and ALI cultures, MUC5AC levels were considerably higher in freshly isolated cells than in ALI cultures. Together this indicates that differentiation of the ALI culture after air exposure induces mainly ciliated cell differentiation with limited goblet cell differentiation (figure 2).

\section{Expression of the non-neuronal cholinergic system}

Next, the expression of genes involved in the non-neuronal cholinergic system in HAE cells was characterised and compared with $16 \mathrm{HBE} 14 \mathrm{o}-$ cells. In view of the rationale of the study, we focused on genes involved in acetylcholine metabolism and muscarinic receptors. Epithelial cells expressed all components of the non-neuronal cholinergic system needed for acetylcholine production and release, as depicted in table 1 for absolute expression levels (Ct values) and in online supplementary table E2 for the relative expression compared with submerged HAE cultures. Choline transporters for uptake of choline were present, including the high-affinity choline transporter 1 (CHT1), the choline transporter-like protein 1 (CTL1), and CTL4. Unexpectedly, the acetylcholine-synthesising enzyme, ChAT, was not detected in any of the epithelial cell cultures, whereas the synthesising enzyme, carnitine acetyltransferase (CarAT), was abundantly expressed. In addition, acetylcholinesterase (AChE) and butyrylcholinesterase (BChE), two enzymes that degrade acetylcholine, were expressed. Finally, muscarinic $\mathrm{M}_{1}, \mathrm{M}_{2}$ and $\mathrm{M}_{3}$ receptors were expressed on the HAE cells as a target for acetylcholine (table 1). Compared with 16HBE14ocells, most components were expressed at similar or higher levels in primary HAE cells. The most prominent higher expression in primary HAE cells was observed for the $M_{3}$ receptor and the choline transporters CHT1 and CTL4 (table 1).

\section{Effect of tiotropium on epithelial cell differentiation}

Since HAE cells express all components of the non-neuronal cholinergic system, suggesting production of endogenous

Table 1 Components of the non-neuronal cholinergic system expressed in epithelial cells

\begin{tabular}{|c|c|c|c|c|}
\hline & $\begin{array}{l}16 \mathrm{HBE} 140- \\
\text { cells }\end{array}$ & $\begin{array}{l}\text { Submerged } \\
\text { cultured } \\
\text { HAE cells }\end{array}$ & $\begin{array}{l}\text { Freshly } \\
\text { isolated } \\
\text { HAE cells }\end{array}$ & $\begin{array}{l}\text { ALI-cultured } \\
\text { HAE cells }\end{array}$ \\
\hline CHT1 & $27.7( \pm 0.5)$ & $24.5( \pm 1.3)$ & $17.1( \pm 0.4)$ & $24.9( \pm 0.7)$ \\
\hline CTL1 & $10.6( \pm 0.3)$ & $12.9( \pm 0.6)$ & $14.3( \pm 0.6)$ & $11.7( \pm 0.5)$ \\
\hline CTL4 & $23.5( \pm 0.4)$ & $17.5( \pm 0.9)$ & $11.9( \pm 0.6)$ & $10.3( \pm 0.7)$ \\
\hline CarAT & $15.5( \pm 1.5)$ & $14.7( \pm 0.8)$ & $14.2( \pm 0.7)$ & $15.3( \pm 0.8)$ \\
\hline AChE & $22.4( \pm 1.0)$ & $17.8( \pm 1.1)$ & $18.5( \pm 1.3)$ & $21.9( \pm 0.8)$ \\
\hline BChE & $19.7( \pm 0.8)$ & $16.5( \pm 0.6)$ & $18.9( \pm 1.3)$ & $19.4( \pm 0.6)$ \\
\hline$M_{1} R$ & $18.7( \pm 1.4)$ & $17.8( \pm 0.7)$ & $17.1( \pm 1.0)$ & $21.2( \pm 0.2)$ \\
\hline$M_{2} R$ & $21.5( \pm 0.5)$ & $19.3( \pm 0.8)$ & $17.5( \pm 0.8)$ & $21.1( \pm 0.4)$ \\
\hline$M_{3} R$ & $25.9( \pm 0.9)$ & $19.8( \pm 1.0)$ & $18.7( \pm 1.4)$ & $17.6( \pm 0.6)$ \\
\hline \multicolumn{5}{|c|}{$\begin{array}{l}\text { Expression of the components was analysed by real-time quantitative PCR in } \\
\text { submerged } 16 \mathrm{HBE} 140-\text { cells and submerged HAE cell cultures, in freshly isolated } \\
\text { HAE cells, and in ALI-cultured HAE cells. Data represent mean ( } \pm \text { SEM) Ct values } \\
\text { corrected for } 18 \mathrm{~S} \text { (ie, highest values represent lowest expression levels), } n=3 \text {. } \\
\text { AChE, acetylcholinesterase; BChE, butyrylcholinesterase; CarAT, carnitine } \\
\text { acetyltransferase; CHT1, high-affinity choline transporter } 1 ; C T L 1 \text {, choline } \\
\text { transporter-like protein } 1 \text {; CTL4, choline transporter-like protein } 4 ; H A E \text {, human } \\
\text { airway epithelial; } M_{1} R \text {, muscarinic } M_{1} \text { receptor; } M_{2} R \text {, muscarinic } M_{2} \text { receptor; } M_{3} R \text {, } \\
\text { muscarinic } M_{3} \text { receptor. }\end{array}$} \\
\hline
\end{tabular}


A

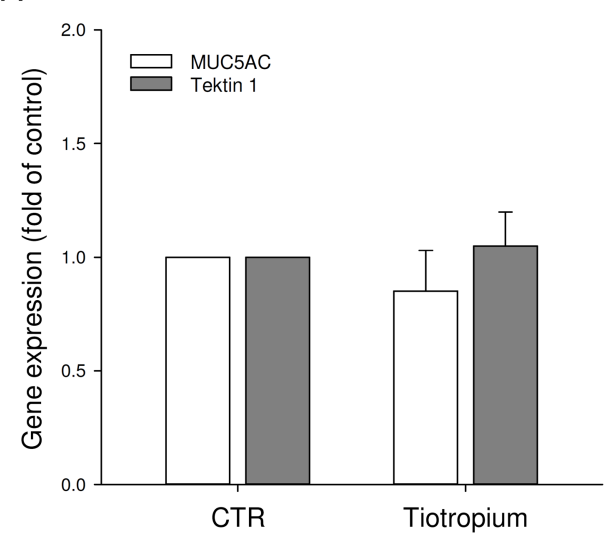

B

CTR

Tiotropium

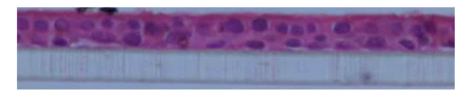

C

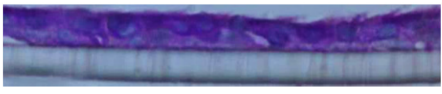

Tiotropium
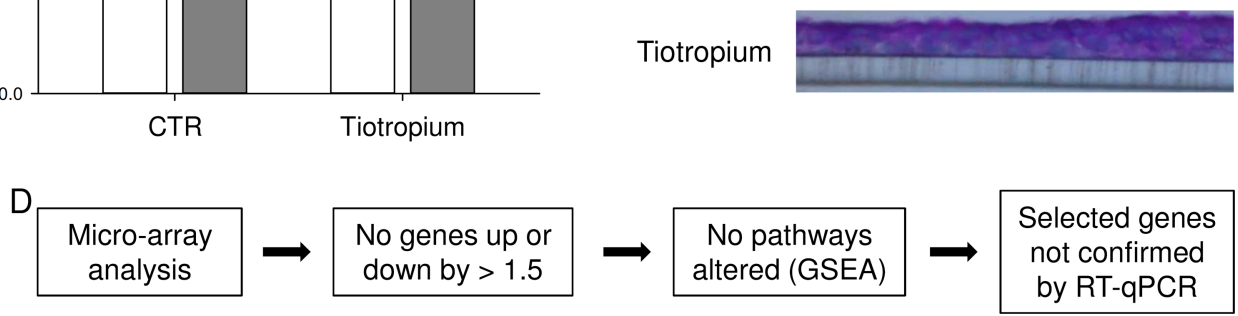

Figure 3 Tiotropium does not affect epithelial cell differentiation after air exposure. Human airway epithelial cells were cultured at an air-liquid interface (ALI) and differentiated with or without tiotropium $(10 \mathrm{nM})$ for 2 weeks as described in figure 1. (A) Expression of the differentiation markers MUC5AC and tektin 1 was analysed by real-time (RT) quantitative (q)PCR. Data represent mean \pm SEM. (B) Representative images of H\&E staining. (C) Representative images of periodic acid Schiff staining. (D) Flow diagram of steps taken after micro-array analysis (four different donors). CTR, control; GSEA, gene set enrichment analysis.

non-neuronal acetylcholine, we examined the effects of the muscarinic antagonist, tiotropium, on epithelial cell differentiation cultured at an ALI. Tiotropium was administered during the 2-week differentiation period at a concentration $(10 \mathrm{nM})$ binding $>99 \%$ of muscarinic receptors. ${ }^{20}$ As depicted in figure $3 \mathrm{~A}$, tiotropium had no effect on gene expression of the differentiation markers MUC5AC and tektin 1 after air exposure. Moreover, no histological changes were observed after exposure to tiotropium, as assessed by H\&E (figure 3B) and PAS (figure 3C) staining. A micro-array analysis confirmed these data and showed that no genes were upregulated or downregulated by more than twofold after treatment with tiotropium, based on cultures derived from four different donors (figure 3D). Gene set enrichment analyses using the KEGG, Reactome and Biocarta definitions revealed no pathways that were significantly altered. In addition, no genes were upregulated or downregulated by more than 1.5 -fold. In an alternative analysis focusing on genes that were upregulated or downregulated by more than 1.1 -fold, 11 genes were selected on the basis of their relevance for epithelial cell differentiation: mitogen-activated protein kinase (MEK) 1, MEK2, MEK4 and rapidly accelerated fibrosarcoma (RAF) 1 of the MAP kinase pathway; laminin (LAM) B3 and LAMC2 involved in cell junction organisation; FoxA2 and FoxA3, involved in goblet cell metaplasia; and the potassium channels KK17, KK15 and KJ6. However, upregulation or downregulation of none of these genes could be reproduced by real-time qPCR using the samples used for micro-array analysis (figure $3 \mathrm{D}$, individual data not shown). Collectively, tiotropium does not affect epithelial cell differentiation after air exposure.

\section{Effect of tiotropium on IL-13-induced goblet cell metaplasia}

Since there was no effect of tiotropium on epithelial cell differentiation after air exposure and, in view of the limited goblet cell differentiation achieved using this method, IL-13 at a concentration of $1 \mathrm{ng} / \mathrm{mL}$ was used in subsequent experiments to induce goblet cell metaplasia. ${ }^{11}$ Stimulation with IL-13 did not affect the expression of receptors and enzymes of the non- neuronal cholinergic system (see online supplementary table E3) and induced only a modest, non-significant increase in inflammatory cytokine expression (see online supplementary table E4). However, stimulation with IL-13 did induce goblet cell metaplasia as assessed by histochemistry and real-time qPCR (figure 4). IL-13 induced a 5.3-fold increase in MUC5ACpositive cells, which was prevented by tiotropium (57\%; figure 4A, B). Moreover, the IL-13-induced increase in goblet cell number, assessed by PAS staining, was completely prevented by tiotropium (figure 4C, D). Furthermore, MUC5AC gene expression was induced by 105 -fold after stimulation with IL-13, which was partly prevented by tiotropium, although this was not significant (47\%; figure 4E). Interestingly, the expression of tektin 1 was decreased by $75 \%$ after stimulation with IL-13 (figure 4F). Tiotropium did not affect the IL-13-induced reduction of tektin 1 (figure 4F).

In subsequent experiments, we investigated the effects of tiotropium on cells exposed to increasing concentrations of IL-13 (1-5 ng/mL). IL-13 induced goblet cell metaplasia in a dose-dependent manner as assessed by immunohistochemistry (figure 5A, B). Interestingly, tiotropium inhibited the increase in MUC5AC-positive cells $(\mathrm{p}=0.012$, two-way analysis of variance, figure $5 \mathrm{~A})$ and goblet cells $(\mathrm{p}=0.015$, two-way analysis of variance, figure $5 \mathrm{~B}$ ).

\section{Effect of tiotropium on established IL-13-induced goblet cell metaplasia}

To further assess the functional relevance of this inhibitory effect of tiotropium, we analysed whether tiotropium was also able to reverse established goblet cell metaplasia. Exposure to IL-13 (1 ng/ $\mathrm{mL}$ ) for 3 weeks induced a 28-fold increase in MUC5AC-positive cells and a 3 -fold increase in goblet cells (figure 6A, B). Treatment with only tiotropium for 3 weeks did not affect MUC5AC or goblet cell number, as observed previously after 2 weeks of exposure. In line with our previous findings, treatment with tiotropium during exposure to IL-13 for 3 weeks prevented IL-13-induced goblet cell metaplasia. Interestingly, tiotropium was also able to 


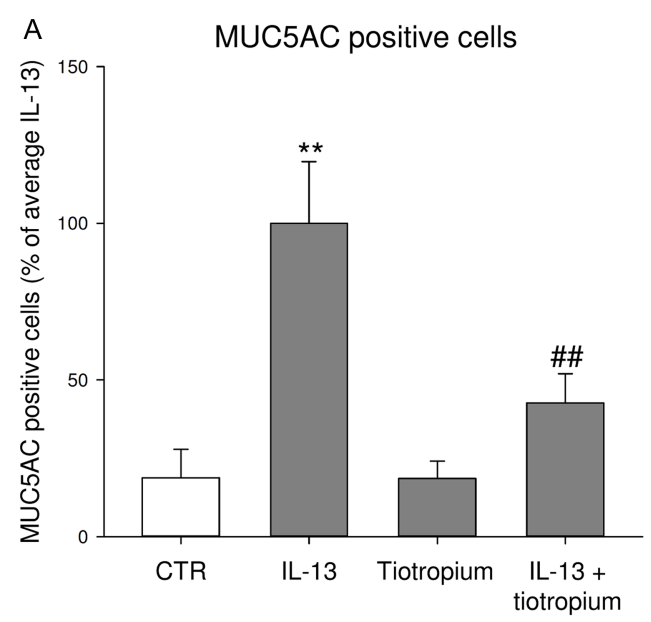

C

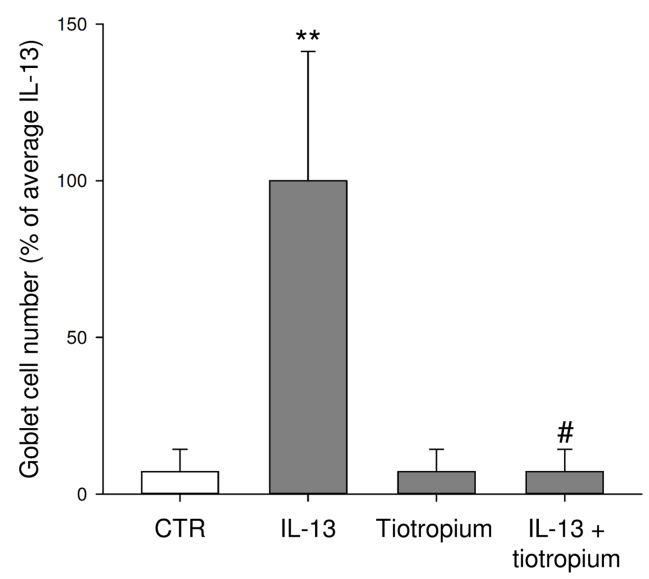

E

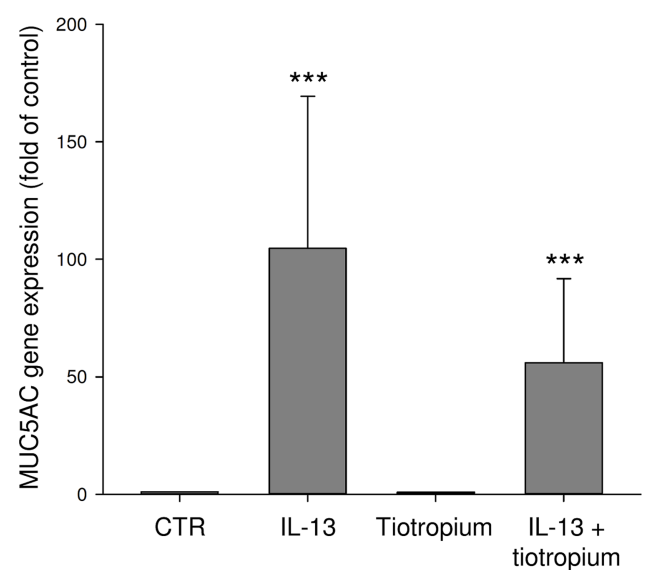

B

CTR

IL-13

Tiotropium

IL-13+ tiotropium
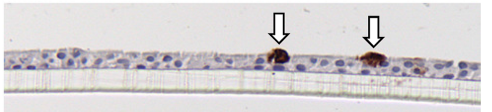

D

CTR

IL-13

Tiotropium

IL-13 + tiotropium

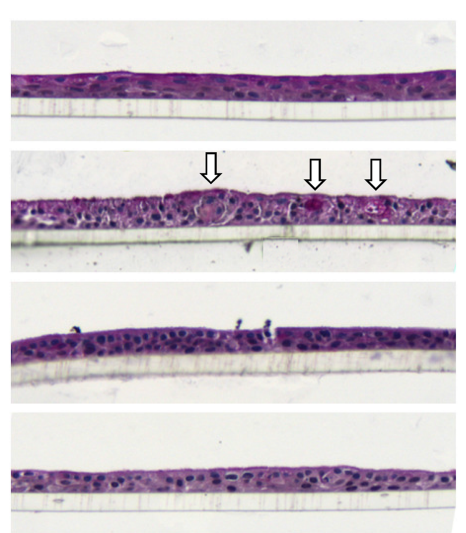

Tektin 1

F

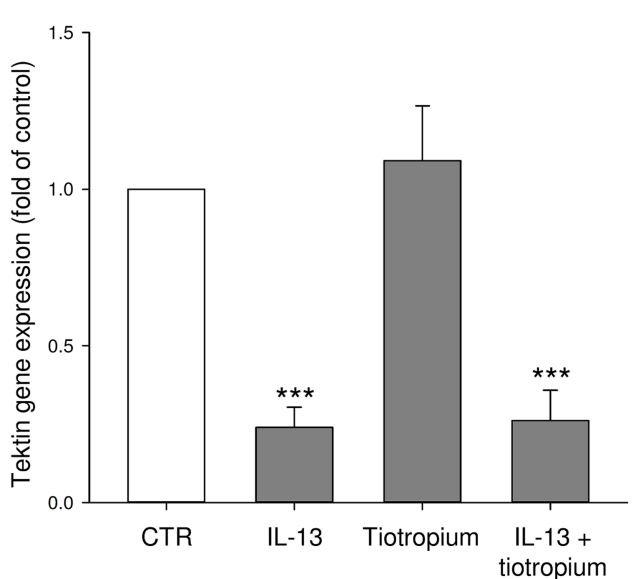

Figure 4 Tiotropium attenuates interleukin (IL)-13-induced goblet cell metaplasia. Human airway epithelial cells were cultured at an air-liquid interface (ALI) and differentiated with or without IL-13 (1 ng/mL) and/or tiotropium (10 nM) for 2 weeks as described in figure 1. MUC5AC-positive cells were determined by MUC5AC antibody staining; quantification $(A)$ and representative images $(B)$ are shown. Goblet cells were determined by periodic acid Schiff staining; quantification (C) and representative images (D) are shown. Expression of the differentiation markers MUC5AC (E) and tektin 1 (F) was analysed by real-time quantitative PCR. CTR, control. Data represent mean $\pm S E M, n=4-8$ donors. ${ }^{* *} p<0.01,{ }^{* * *} p<0.001$ compared with CTR; \#p<0.05, \#\#p<0.01 compared with IL-13.

reverse established goblet cell metaplasia, as it inhibited the increase in MUC5AC-positive cells (90\%, figure 6A) and the increase in goblet cells $(50 \%$, figure $6 \mathrm{~B})$ when tiotropium was applied in the last week only in combination with 3 weeks of continued stimulation with IL-13.

\section{Transcriptional mechanisms involved}

In subsequent experiments, we investigated the key transcription factors involved in IL-13-induced goblet cell metaplasia in HAE cells and the effect of tiotropium thereon. As depicted in figure 7, increased MUC5AC expression was accompanied by decreased 
A

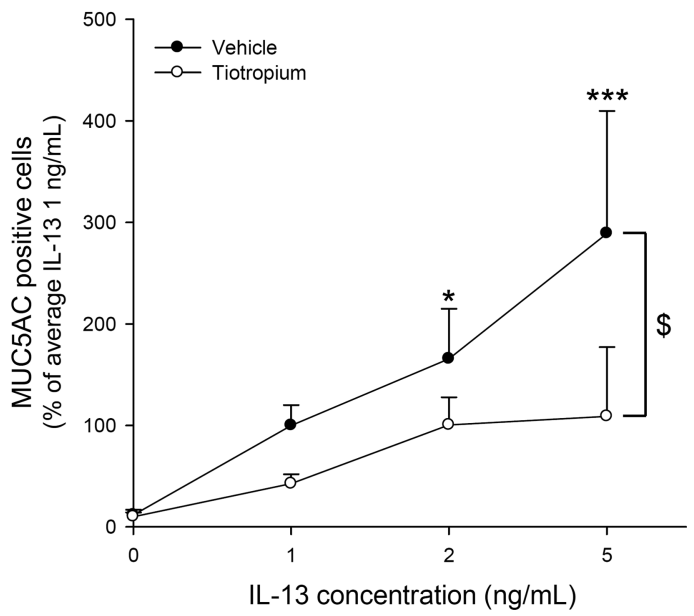

B

Goblet cells

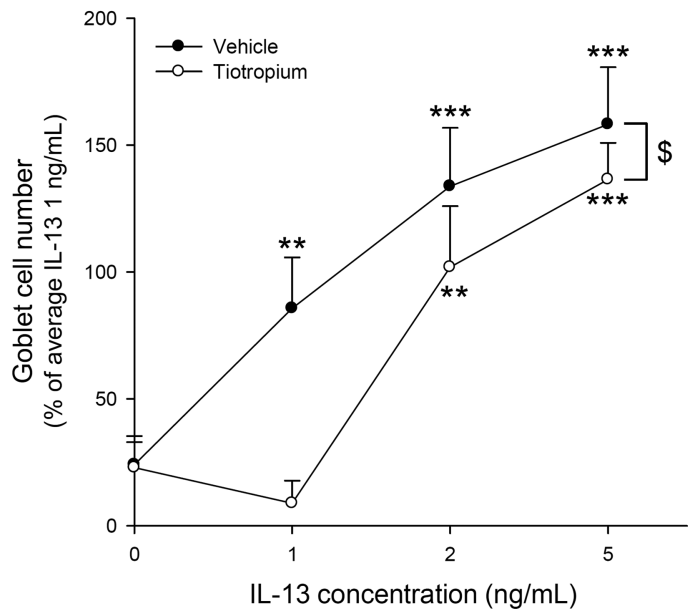

Figure 5 Tiotropium attenuates interleukin (IL)-13-induced goblet cell metaplasia in a dose-dependent manner. Human airway epithelial cells were cultured at an air-liquid interface (ALI) and differentiated with or without IL-13 (1, 2 or $5 \mathrm{ng} / \mathrm{mL})$ and/or tiotropium (10 nM) for 2 weeks as described in figure 1. MUC5AC-positive cells were determined by MUC5AC antibody staining (A), and goblet cells were determined by periodic acid Schiff staining (B). CTR, control. Data represent mean $\pm S E M, n=4$ donors. $\$ p<0.05$ for treatment effect; ${ }^{*} p<0.05$, ${ }^{* *} p<0.01$, ${ }^{* * *} p<0.001$ compared with CTR.

expression of FoxA2 (1.6-fold) and increased expression of FoxA3 (3.6-fold) and SPDEF (5.2-fold). Nuclear localisation of FoxA2 was demonstrated by immunohistochemistry (data not shown). Tiotropium had no effect on basal expression of these genes, but completely reversed the inhibitory effect of IL-13 on FoxA2 (figure 7A). Moreover, tiotropium partially prevented IL-13induced FoxA3 expression (figure 7B). IL-13-induced SPDEF expression was not affected by tiotropium (figure $7 \mathrm{C}$ ).

\section{DISCUSSION}

In this study we demonstrate that non-neuronal acetylcholine contributes to goblet cell differentiation. HAE cells expressed all components needed for acetylcholine synthesis, and, although the muscarinic antagonist tiotropium did not affect epithelial cell differentiation after air exposure, tiotropium did inhibit IL-13-induced goblet cell metaplasia. In addition, tiotropium was able to reverse established IL-13-induced goblet cell metaplasia. Furthermore, we provide evidence for the involvement of the transcriptional regulators, FoxA2 and FoxA3, in this effect. This study is the first to investigate the role of tiotropium in ALI-cultured primary epithelial cells, and the results imply that tiotropium might affect mucus hypersecretion.

Data from this study clearly indicate that all components needed for acetylcholine synthesis are present in cultured HAE cells derived from central airways. The fact that epithelial cells express components of the non-neuronal cholinergic system is in line with previous studies. ${ }^{5}{ }^{6}$ However, in contrast with previous studies and different from the neuronal cholinergic system where ChAT is the most prominent enzyme of acetylcholine synthesis, ${ }^{4}{ }^{6}$ we were unable to detect significant expression of ChAT in any of the epithelial cell cultures. Rather, we demonstrate abundant expression of the acetylcholine-synthesising
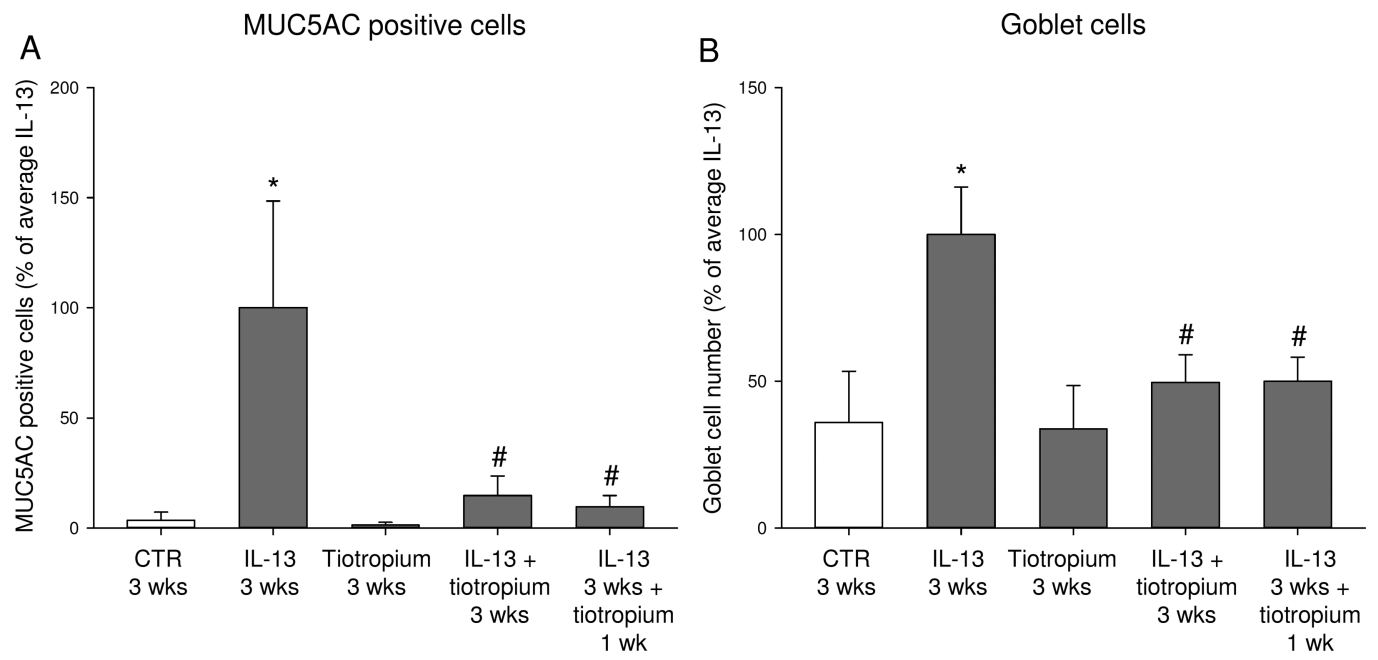

Figure 6 Tiotropium reverses established goblet cell metaplasia induced by interleukin (IL)-13. Human airway epithelial cells were cultured at an air-liquid interface (ALI) and differentiated with or without IL-13 $(1 \mathrm{ng} / \mathrm{mL})$ and/or tiotropium $(10 \mathrm{nM})$ for 2 weeks. Thereafter, cells were cultured for an additional week with IL-13 and/or tiotropium as described in figure 1. MUC5AC-positive cells were determined by MUC5AC antibody staining (A), and goblet cells were determined by periodic acid Schiff staining (B). CTR, control; wk, week. Data represent mean $\pm S E M, n=4$ donors. ${ }^{*} p<0.05$ compared with CTR; \#p<0.05 compared with IL-13. 
Figure 7 Effect of tiotropium on transcriptional mechanisms involved in goblet cell metaplasia. Human airway epithelial cells were cultured at an airliquid interface (ALI) and differentiated with or without interleukin (IL)-13 (1 $\mathrm{ng} / \mathrm{mL}$ ) and/or tiotropium (10 nM) for 2 weeks as described in figure 1 . Expression of the transcription factors forkhead box protein A2 (FoxA2; A), forkhead box protein A3 (FoxA3; B) and SAM-pointed domain-containing ETS transcription factor (SPDEF; C) was analysed by real-time quantitative PCR. $\mathrm{CTR}$, control. Data represent mean \pm SEM, $n=8$ donors. ${ }^{*} p<0.05$, CTR; \#p<0.05 compared with IL-13. ${ }^{* *} p<0.01,{ }^{* * *} p<0.001$ compared with

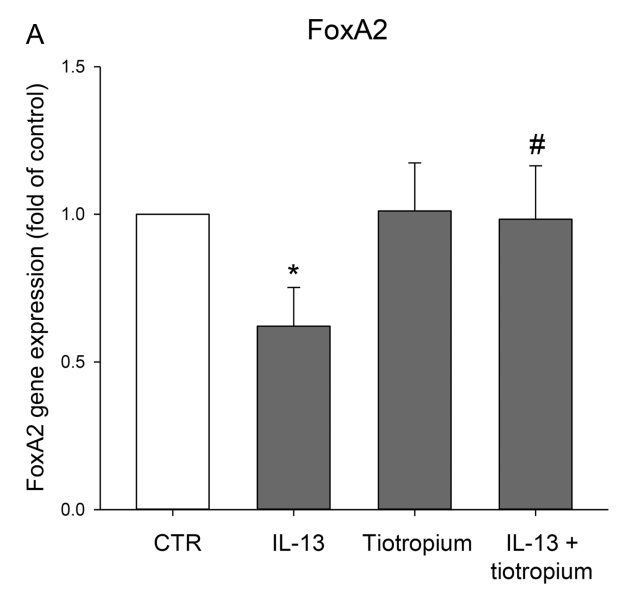

B FoxA3
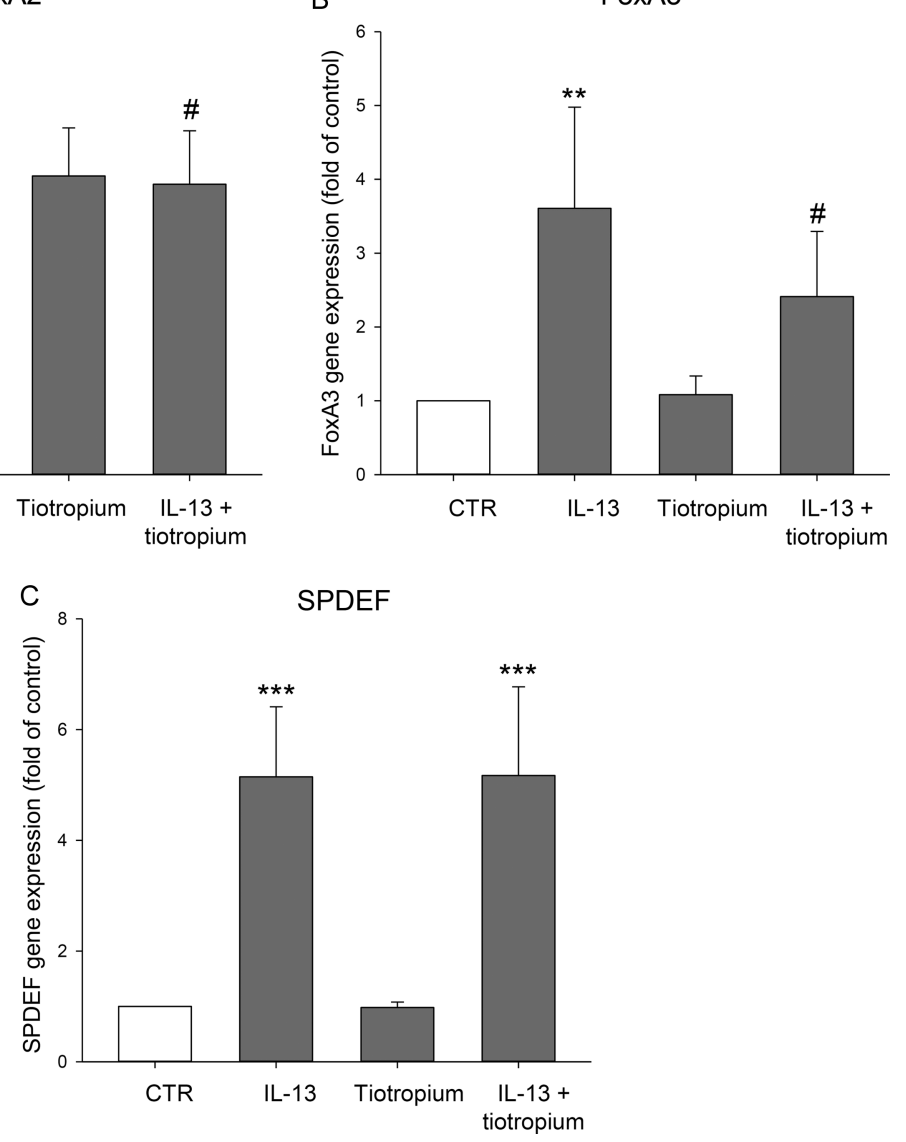

enzyme, CarAT. CarAT was first identified 40 years ago as an alternative way to synthesise acetylcholine in rabbit heart by White and $\mathrm{Wu}^{21}$ and has been detected in multiple nonneuronal cholinergic systems since then, including rat skeletal muscle, ${ }^{22}$ cat oesophageal mucosa, ${ }^{23}$ mouse bladder and abraded urothelium, and human mucosal bladder biopsies. ${ }^{24}$

The fact that acetylcholine is both a neurotransmitter and a local signalling molecule produced by non-neuronal cells has dramatically broadened the view on the role of acetylcholine. It has been suggested that the non-neuronal cholinergic system might play a role in lung physiology and/or pathophysiology, but direct evidence for such a role is still limited. In this study, HAE cells cultured at an ALI were used as a model system. In this way, the role of non-neuronal acetylcholine can be investigated, since no neuronal system is present. Moreover, by using primary cells cultured at an ALI, human physiology is reflected more closely than by using cell lines such as 16HBE14o-. Instead of only one basal cell type, an ALI culture is a pseudostratified mucociliary cell culture with different epithelial cells present, as is also observed in vivo. Our data indicate that ciliated cell marker expression in ALI-cultured HAE cells is comparable to that in freshly isolated airway epithelial cells, whereas MUC5AC expression, a marker for goblet cells, is moderately expressed after ALI culture, but can be markedly increased by IL-13 exposure. Of specific relevance to our study is the finding that components of the non-neuronal cholinergic system are expressed at higher levels in primary ALI-cultured cells than in 16HBE14o- cells.

Tiotropium treatment inhibited IL-13-induced goblet cell differentiation, but did not affect epithelial cell differentiation after air exposure. Based on $\mathrm{n}=4$ in duplicate, an SD per gene of 0.5 and an expected fold change of 2 , a power of 0.8 can be calculated assuming a false positive rate of 1 in $20(5 \%)$ genes in the microarray study. No genes were found significantly up- or downregulated in this study, even when gene sets were taken into consideration (KEGG, Reactome and Biocarta gene sets). Although the study was not powered for this, when lowering the fold change to 1.1 , some gene changes were detected with a $\mathrm{p}<0.05$. However, none of these were confirmed in subsequent qPCR studies. Therefore, we believe that tiotropium does not affect epithelial cell differentiation in cultures not exposed to additional stimuli, but does affect IL-13-induced goblet cell differentiation.

IL-13 is a central mediator in asthma pathology leading to goblet cell metaplasia and mucus hypersecretion. ${ }^{8}$ Mucus secretion and MUC5AC expression are enhanced in asthma and contribute significantly to airflow limitation in asthma by obstructing the smaller airways. ${ }^{25} 26$ Stimulation with IL-13 increased MUC5AC expression in a dose-dependent manner in our study, which is in line with previous studies using ALI cultures. ${ }^{1011}$ Moreover, expression of tektin 1, a marker of ciliated cells, was reduced at the same time by IL-13. Indeed goblet cell metaplasia was induced, as MUC5AC antibody staining and PAS staining revealed an increase in mucin-producing cells compared with control sections. Furthermore, expression of the transcription factors SPDEF and FoxA3 was enhanced, whereas expression of FoxA2 was reduced. This is in line with previous studies aimed at unravelling the transcriptional network involved in goblet cell metaplasia. ${ }^{12}{ }^{13}$ Interestingly, tiotropium completely prevented the reduced expression of FoxA2 and partly prevented the increase in FoxA3, whereas increased expression of SPDEF was not affected. This may explain the partial effect observed on goblet cell metaplasia after tiotropium exposure. 
Mucus-secreting cells in the airways include epithelial goblet cells and submucosal glands. It has been convincingly shown that the latter are under cholinergic neural control. ${ }^{25}$ Vagal stimulation of ferret trachea in vitro or in vivo enhanced mucus secretion, which was inhibited by the $\mathrm{M}_{3}$ antagonist, 4-DAMP, ${ }^{27}$ and electrical field stimulation also enhanced mucus release from human bronchi. ${ }^{28}$ This neuronal acetylcholine-induced mucus release is probably derived from airway submucosal glands. However, there is increasing evidence that acetylcholine can also induce mucus secretion from goblet cells. ${ }^{25} 2930$ Whether goblet cells can release mucus in response to neuronally released acetylcholine is still a matter of debate, but our data do suggest a role for non-neuronal acetylcholine in the differentiation towards this cell type. While submucosal glands predominate in healthy human airways, goblet cells undergo metaplasia under disease conditions and predominate in asthma, ${ }^{25}$ indicating the potential relevance for inhibition of this differentiation process.

Therefore, the finding that tiotropium partly prevents IL-13-induced goblet cell metaplasia in our model implies that this might be beneficial for patients with asthma. This is in line with evidence from in vitro and in vivo studies indicating a role for muscarinic antagonists in mucus hypersecretion. The muscarinic antagonist, oxitropium, dose-dependently inhibits histamine-induced mucus secretion from airway goblet cells. ${ }^{31}$ Furthermore, Cortijo et $a l^{32}$ demonstrated that muscarinic receptor stimulation by carbachol induced an increase in MUC5AC expression in human bronchus and in cultured epithelial cells, which was inhibited by the muscarinic antagonist, aclidinium. Our data indicate that tiotropium is also able to reverse established goblet cell hyperplasia, which is of interest and importance. This suggests that the turnover of goblet cells is sufficiently rapid to allow reversal of this phenotype within 1 week and argues that cholinergic pressure in combination with IL-13 is required to both induce and maintain a differentiated goblet cell phenotype. We have to consider that all these studies, including the study presented in this paper, used healthy cells to investigate the effects of muscarinic receptor stimulation on goblet cell metaplasia. Intriguingly, however, similar findings were observed in patients with mild asthma, in whom repeated challenges with methacholine induced epithelial cell proliferation and goblet cell metaplasia. ${ }^{17}$ Interestingly, these effects can be prevented when bronchoconstriction is prevented, suggesting that preventing bronchoconstriction with tiotropium might have an indirect inhibitory effect on mucus hypersecretion. ${ }^{17}$ Animal studies have also provided indirect evidence of a role for muscarinic receptors in allergen-induced goblet cell metaplasia. In an ovalbumin-induced guinea pig model, tiotropium inhibited MUC5AC expression and mucus gland hypertrophy. ${ }^{14}$ Moreover, goblet cell metaplasia was inhibited by tiotropium in an ovalbumin-induced mouse model. ${ }^{15}$ Interestingly, knockout of the muscarinic M3 receptor also inhibits ovalbumin-induced goblet cell metaplasia and MUC5AC expression. ${ }^{16}$ Notably, IL-13 expression was enhanced in the same animals, indicating that this effect was not mediated via a reduction of the inflammatory response. ${ }^{16}$

Our findings extend these previous observations, as we now demonstrate that the effects of tiotropium are not only the result of reduced bronchoconstriction or reduced inflammatory cytokine expression, but that tiotropium also has a direct effect on the epithelium. We performed a multiplex cytokine analysis to verify the absence of the involvement of an inflammatory response. Only a modest, non-significant increase in inflammatory cytokines was observed after stimulation with IL-13, and, furthermore, tiotropium had no significant effect on inflammatory cytokines. Therefore we can conclude from our study that airway epithelial cells express non-neuronal acetylcholine, and inhibition of this component by anticholinergic agents is sufficient to inhibit IL-13-induced goblet cell differentiation. This direct effect of tiotropium on airway epithelial cells as observed in our study might act synergistically with indirect effects of tiotropium on epithelial cells in vivo, including reduced mechanical strain and reduced inflammation. However, future clinical studies are needed to confirm this hypothesis.

Tiotropium is currently registered as a chronic therapy for patients with severe asthma. Recent evidence indicates that treatment with tiotropium induces bronchodilation in patients with moderate and severe asthma. ${ }^{133}$ In patients with severe asthma, use of tiotropium on top of standard therapy with long-acting $\beta$-agonists and inhaled corticosteroids both induces an increase in $\mathrm{FEV}_{1}$ and reduces the number of severe exacerbations and episodes of worsening of asthma. ${ }^{2}$ Results from our study imply that mucus hypersecretion might also be affected by tiotropium treatment. Previously, tiotropium has been shown to be effective in reducing sputum levels in patients with chronic airway mucus hypersecretion. ${ }^{34}$ Although it has been proposed that anticholinergic treatment might impair sputum clearance, there is now increasing evidence that reducing the volume of sputum without altering viscoelasticity with anticholinergic agents is beneficial for patients with chronic mucus hypersecretion. ${ }^{35}$ Future studies are needed to directly address this hypothesis in more detail.

\section{CONCLUSIONS}

We demonstrate that tiotropium can prevent, and also reverse, IL-13-induced goblet cell metaplasia, indicating that nonneuronal acetylcholine contributes to goblet cell differentiation. Modulation of FoxA2 and FoxA3 expression is probably one of the mechanisms involved. These data imply that this direct effect of tiotropium on the airway epithelium, in combination with its protective effects on bronchoconstriction and inflammation, may protect against mucus hypersecretion.

Acknowledgements The authors thank Renate M Verhoosel and Willemieke $M$ Mudde for technical assistance, and Tinne C J Mertens for expert advice on analysis of IL-13-induced mucin production. This study was financially supported by the Netherlands Lung Foundation (grant 3.2.08.014). Previous research on the ALI model in the laboratory of P S Hiemstra was supported by a grant from Boerhringer Ingelheim.

Contributors LEMK conceived the study, participated in its design, carried out the study and drafted the manuscript. STB and SB carried out part of the study. PSH participated in the study design and setting up the ALI culture. MB carried out the micro-array analysis. MNH, HAMK and HM participated in the study design. RG conceived the study, participated in its design and coordination and helped to draft the manuscript. All authors revised the manuscript critically for important intellectual content and approved the final version of the manuscript.

Funding This study was financially supported by the Netherlands Lung Foundation (grant 3.2.08.014).

Competing interests PSH, HAMK, HM and RG received research grants from Boehringer Ingelheim.

Provenance and peer review Not commissioned; externally peer reviewed.

\section{REFERENCES}

1 Kerstjens HA, Casale TB, Bleecker ER, et al. Tiotropium or salmeterol as add-on therapy to inhaled corticosteroids for patients with moderate symptomatic asthma: two replicate, double-blind, placebo-controlled, parallel-group, active-comparator, randomised trials. Lancet Respir Med 2015;3:367-76.

2 Kerstjens HA, Engel M, Dahl R, et al. Tiotropium in asthma poorly controlled with standard combination therapy. N Engl J Med 2012;367(Suppl 13):1198-207.

3 Wessler I, Kirkpatrick CJ. Acetylcholine beyond neurons: the non-neuronal cholinergic system in humans. Br J Pharmacol 2008;154(Suppl 8):1558-71. 
4 Klapproth H, Reinheimer T, Metzen J, et al. Non-neuronal acetylcholine, a signalling molecule synthesized by surface cells of rat and man. Naunyn Schmiedebergs Arch Pharmacol 1997;355(Suppl 4):515-23.

5 Proskocil BJ, Sekhon HS, Jia Y, et al. Acetylcholine is an autocrine or paracrine hormone synthesized and secreted by airway bronchial epithelial cells. Endocrinology 2004;145(Suppl 5):2498-506.

6 Kummer W, Lips KS, Pfeil U. The epithelial cholinergic system of the airways. Histochem Cell Biol 2008;130(Suppl 2):219-34.

7 Lambrecht BN, Hammad H. The airway epithelium in asthma. Nat Med 2012;18 (Suppl 5):684-92

8 Corren J. Role of interleukin-13 in asthma. Curr Allergy Asthma Rep 2013; 13(Suppl 5):415-20.

9 Zhu Z, Homer RJ, Wang Z, et al. Pulmonary expression of interleukin-13 causes inflammation, mucus hypersecretion, subepithelial fibrosis, physiologic abnormalities, and eotaxin production. J Clin Invest 1999;103(Suppl 6):779-88.

10 Laoukili J, Perret E, Willems T, et al. IL-13 alters mucociliary differentiation and ciliary beating of human respiratory epithelial cells. J Clin Invest 2001;108(Suppl 12):1817-24.

11 Zuyderduyn S, Ninaber DK, Schrumpf JA, et al. IL-4 and IL-13 exposure during mucociliary differentiation of bronchial epithelial cells increases antimicrobial activity and expression of antimicrobial peptides. Respir Res 2011;12:59.

12 Whitsett JA, Haitchi HM, Maeda Y. Intersections between pulmonary development and disease. Am J Respir Crit Care Med 2011;184(Suppl 4):401-6.

13 Chen G, Korfhagen TR, Xu Y, et al. SPDEF is required for mouse pulmonary goblet cell differentiation and regulates a network of genes associated with mucus production. J Clin Invest 2009;119(Suppl 10):2914-24.

14 Bos IS, Gosens R, Zuidhof $A B$, et al. Inhibition of allergen-induced airway remodelling by tiotropium and budesonide: a comparison. Eur Respir J 2007;30 (Suppl 4):653-61

15 Ohta S, Oda N, Yokoe T, et al. Effect of tiotropium bromide on airway inflammation and remodelling in a mouse model of asthma. Clin Exp Allergy 2010; 40(Suppl 1365-2222; 0954-7894; 8):1266-75.

16 Kistemaker LE, Bos ST, Mudde WM, et al. Muscarinic m3 receptors contribute to allergen-induced airway remodeling in mice. Am J Respir Cell Mol Biol 2014;50 (Suppl 4):690-8.

17 Grainge CL, Lau LC, Ward JA, et al. Effect of bronchoconstriction on airway remodeling in asthma. N Engl J Med 2011;364(Suppl 21):2006-15.

18 Cozens AL, Yezzi MJ, Kunzelmann K, et al. CFTR expression and chloride secretion in polarized immortal human bronchial epithelial cells. Am J Respir Cell Mol Biol 1994;10(Suppl 1):38-47.
19 Gkoumassi E, Dekkers BG, Droge MJ, et al. Virodhamine and CP55,940 modulate CAMP production and IL-8 release in human bronchial epithelial cells. $\mathrm{Br} J$ Pharmacol 2007;151(Suppl 7):1041-8.

20 Disse B, Speck GA, Rominger KL, et al. Tiotropium (Spiriva): mechanistical considerations and clinical profile in obstructive lung disease. Life Sci 1999;64(Suppl 6-7):457-64.

21 White $\mathrm{HL}, \mathrm{Wu} J \mathrm{C}$. Choline and carnitine acetyltransferases of heart. Biochemistry 1973;12(Suppl 5):841-6.

22 Tucek $S$. The synthesis of acetylcholine in skeletal muscles of the rat. J Physiol 1982;322:53-69.

23 Wolf-Johnston AS, Hanna-Mitchell AT, Buffington CA, et al. Alterations in the non-neuronal acetylcholine synthesis and release machinery in esophageal epithelium. Life Sci 2012;91(Suppl 21-22):1065-9.

24 Lips KS, Wunsch J, Zarghooni S, et al. Acetylcholine and molecular components of its synthesis and release machinery in the urothelium. Eur Urol 2007;51(Suppl 4):1042-53.

25 Rogers DF. Motor control of airway goblet cells and glands. Respir Physiol 2001;125(Suppl 1-2):129-44.

26 Morcillo EJ, Cortijo J. Mucus and MUC in asthma. Curr Opin Pulm Med 2006;12 (Suppl 1):1-6.

27 Ramnarine $\mathrm{SI}$, Haddad EB, Khawaja AM, et al. On muscarinic control of neurogenic mucus secretion in ferret trachea. J Physio/ 1996;494(Pt 2)(Suppl Pt 2):577-86.

28 Baker B, Peatfield AC, Richardson PS. Nervous control of mucin secretion into human bronchi. J Physiol 1985;365:297-305.

29 Bolduc $P$, Reid $\mathrm{L}$. The effect of isoprenaline and pilocarpine on mitotic index and goblet cell number in rat respiratory epithelium. Br J Exp Pathol 1978;59(Suppl 3):311-18.

30 Steel DM, Hanrahan JW. Muscarinic-induced mucin secretion and intracellular signaling by hamster tracheal goblet cells. Am J Physiol 1997;272(2 Pt 1):L230-7.

31 Takeyama K, Tamaoki J, Nakata J, et al. Effect of oxitropium bromide on histamine-induced airway goblet cell secretion. Am J Respir Crit Care Med 1996;154(Suppl 1):231-6.

32 Cortijo J, Mata M, Milara J, et al. Aclidinium inhibits cholinergic and tobacco smoke-induced MUC5AC in human airways. Eur Respir J 2011;37(Suppl 2):244-54.

33 Kerstjens HA, Disse B, Schroder-Babo W, et al. Tiotropium improves lung function in patients with severe uncontrolled asthma: a randomized controlled trial. J Allergy Clin Immunol 2011;128(Suppl 2):308-14.

34 Saito Y, Azuma A, Morimoto T, et al. Tiotropium ameliorates symptoms in patients with chronic airway mucus hypersecretion which is resistant to macrolide therapy. Intern Med 2008;47(Suppl 7):585-91.

35 Bateman ED, Rennard S, Barnes PJ, et al. Alternative mechanisms for tiotropium. Pulm Pharmacol Ther 2009;22(Suppl 6):533-42. 